\title{
Separation selectivity of some phenolic acids in RP HPLC systems with binary mobile phase comprised various modifiers
}

\author{
Anna Klimek-Turek • Tadeusz H. Dzido
}

Received: 5 March 2010 / Accepted: 31 May 2010 / Published online: 30 June 2010

(C) The Author(s) 2010. This article is published with open access at Springerlink.com

\begin{abstract}
Retention of phenolic acid has been correlated for reversed-phase high-performance liquid chromatography systems with different binary mobile phases containing methanol, acetonitrile or tetrahydrofuran as modifiers and buffer at $\mathrm{pH}$ 3.0, 4.6 and 5.0. The changes of separation selectivity of solutes, when one modifier is replaced by another in the eluent, has been explained taking into consideration molecular interactions of the solutes with components of the stationary phase region, i.e. extracted modifier, and ordering of the stationary phase by the modifier.
\end{abstract}

Keywords Modifier selectivity · Mechanism of separation selectivity in reversed phase liquid chromatography .

Phenolic acids

\section{Introduction}

Liquid chromatography is the most popular mode for analytical separations of chemical compounds in pharmaceutical, biomedical and environmental pollution samples. Optimization of chromatographic conditions is the most important task of performing separation of mixture components. Typical values of retention factor of the solutes should be in the range 1-10 and values of resolution greater than 1.5 when quantitative analysis to be performed. Separation selectivity can be varied with type of the stationary and the mobile phases (Szepesy 2002; Lavine et al. 2002; Sandi and Szepesy 1999). However, the simplest way leading very often to a variation of separation selectivity of

A. Klimek-Turek · T.H. Dzido $(\bowtie)$

Department of Physical Chemistry, Medical University of Lublin, ul. Staszica 6, 20-081 Lublin, Poland

e-mail: tadeusz.dzido@umlub.pl solutes is based on alteration of composition of the mobile phase including concentration and type of a modifier (organic component of the mobile phase) and $\mathrm{pH}$ of buffer. The last variable is especially valuable when solutes are weak electrolytes. If solutes do not undergo dissociation then, in general, $\mathrm{pH}$ variation does not lead to selectivity change. However, with the possible exception of the solutes, which demonstrate proton acceptor properties because proton donor property of silanol groups of the stationary phase is altered with $\mathrm{pH}$ change of the mobile phase. Replacement of the modifier type in the mobile phase can lead to an alteration of separation selectivity. A choice of the appropriate solvent is often an real challenge, especially if one takes into account many possible molecular interactions of separated solutes with components of the mobile and stationary phases.

In our previous paper we proposed the approach in which separation selectivity changes can be explained by molecular interactions of solute molecules and components of the stationary phase. This approach was presented in the paper (Dzido 2000). Basing on the data of partition constants of benzene derivatives with various polar groups for gas-liquid systems, where liquid solutions were binary solvents comprised water and methanol $(\mathrm{MeOH})$, acetonitrile $(\mathrm{ACN})$ or tetrahydrofuran (THF). It was concluded that partition selectivity was not dependent on organic solvent type. Such conclusion has been used to express the hypothesis that changes of separation selectivity of solutes in reversed-phase systems, with eluents of similar qualitative and quantitative composition as in gas-liquid systems mentioned, do not originate from the mobile phase when modifier is replaced in it. In this way the separation selectivity changes caused by replacement of the modifier in the mobile phase could be explained by molecular interactions of the solutes in the stationary phase. In addition this approach was confirmed tak- 
ing into account properties of reversed-phase system with silica based stationary phases of C8 and C18 type (Dzido 2000; Dzido et al. 2002). Application of this approach to explanation of separation selectivity changes was demonstrated in the paper (Dzido et al. 2002) for aromatic hydrocarbons with various polar groups separated in reversedphase systems with stationary phase of C18 type. It should be mentioned that this approach seems to be advantageous from one more reason. It eliminates ambiguity concerned with molecular interactions of solutes in both mobile and stationary phases. E.g. retention increase of compound 1 relative to compound 2 , when modifier $\mathrm{A}$ is replaced with modifier $\mathrm{B}$ in the mobile phase, could be explained by enhancement of molecular interactions of compound 1 relative to compound 2 in the stationary phase and/or diminution of these interactions in the mobile phase. Our approach of explanation of selectivity changes, when one mobile phase modifier is replaced by another, is considerably simplified because molecular interactions of the solutes with the stationary phase components are considered only.

In reversed-phase liquid chromatography systems a silica based stationary phase of the C18 type comprises following components: aliphatic $\mathrm{C} 18$ ligands, free silanols, water and mobile phase modifier (organic solvent). Quantity of the two first components is constant. Concentration of water in the stationary phase region also approaches approximately constant value in restricted its concentration range in the mobile phase. So the modifier is that component, which is mainly responsible for difference between properties of the stationary phases in systems with various organic solvents in the mobile phase, compare discussion in (Dzido et al. 2002). The three organic solvents, methanol, acetonitrile and tetrahydrofuran, are the most often applied in practice of RP HPLC (reversed-phase high-performance liquid chromatography). These modifiers demonstrate strong extraction into the stationary phase of C18 type in reversed phase systems (McCormick and Karger 1980; Slaats et al. 1981; Gilpin et al. 1990; Kazakevich et al. 2001; Bocian et al. 2009). This extraction increases in the order $\mathrm{MeOH}, \mathrm{ACN}$ and THF. However, ability of these modifiers to molecular interactions is quite different. Methanol is characterized by high contribution to interact as proton donor, possesses a strong proton acceptor and relative high dipolar properties. Acetonitrile possesses the largest dipole moment among the modifiers but is characterized by weak property to be a proton acceptor and much weaker as a proton donor. The electric dipole moment of acetonitrile is almost two times greater than that of tetrahydrofuran (3.45 D and 1.75 D for acetonitrile and tetrahydrofuran, respectively) (Karapetian and Eichis 1989; Bidlingmeyer 1980). Tetrahydrofuran shows no ability to be a proton donor, is characterized by a stronger proton acceptor property than acetonitrile, but its ability to dipolar interactions is lower.
Table 1 Kamlet-Taft solvatochromic parameters

\begin{tabular}{llll}
\hline & $\begin{array}{l}\text { Hydrogen } \\
\text { bond acidity } \\
(\alpha)\end{array}$ & $\begin{array}{l}\text { Hydrogen } \\
\text { bond basicity } \\
(\beta)\end{array}$ & $\begin{array}{l}\text { Dipolarity } \\
(\pi)\end{array}$ \\
\hline Methanol & 0.93 & 0.62 & 0.60 \\
Acetonitrile & 0.19 & 0.31 & 0.75 \\
Tetrahydrofuran & 0.00 & 0.55 & 0.58 \\
\hline
\end{tabular}

In Table 1 the mentioned properties of the modifiers are reflected in the values of their solvatochromic parameters $(\alpha, \beta$ and $\pi$ ) (Abraham et al. 1999; Kiridena and Poole 1998; Tan et al. 1996). Hydrogen bond basicity, $\beta$, is equal to $0.55,0.31$ and 0.62 for THF, $\mathrm{ACN}$ and $\mathrm{MeOH}$, respectively. Hydrogen bond acidity, $\alpha$, is equal to $0.00,0.19$ and 0.93 for THF, ACN and $\mathrm{MeOH}$, respectively. Property of these modifiers to dipolar interactions is characterized by parameter $\pi$, of which values are equal to $0.58,0.75$ and 0.60 for THF, $\mathrm{ACN}$ and $\mathrm{MeOH}$, respectively. The values of refraction indice of THF, $\mathrm{ACN}$ and $\mathrm{MeOH}$ are equal to $1.405,1.342,1.329$, respectively. These values indicate higher property of THF to dispersive interactions than that of $\mathrm{ACN}$ and $\mathrm{MOH}$.

Taking into account the mentioned properties of tetrahydrofuran, and due to its almost planar molecular structure and higher molecular volume (and surface) in comparison to acetonitrile and methanol, the stationary phase region in THF systems is more ordered than that in ACN one and considerably stronger than in $\mathrm{MeOH}$ system.

As it was mentioned above our approach, explanation of selectivity changes by molecular interactions of the solutes with stationary phase components (especially modifier) when mobile phase modifier is replaced with another one, had been successfully tested for aromatic hydrocarbons with polar groups (Dzido et al. 2002). In the paper we intend to extend our investigation to other group of solutes, which undergo dissociation. We have chosen phenolic acids to research into this problem.

\section{Experimental}

Solutes (phenolic acids, Table 2) were obtained from different sources. All solvents (methanol, acetonitrile and tetrahydrofuran) were analytical grade. Water was bidistilled. The mobile phase comprised acetic acid buffer $(0.02 \mathrm{M})$ at $\mathrm{pH}$ 3.0, 4.6 and 5.0. Measurements of retention of phenolic acids were performed with a HP 1050 liquid chromatograph (Hewlett-Packard, Palo Alto, CA, USA) equipped with $20 \mu \mathrm{L}$ sample injector (Rheodyne, Cotati, California, USA) and a variable UV detector (HP-1050) operating at 254 $\mathrm{nm}$. Chromatograph was equipped with a Zorbax SB C-18 (4.6 $\mathrm{mm} \times 150 \mathrm{~mm}, 5 \mu \mathrm{m}$, Agilent Technologies) column. 
Table 2 Abraham's solvation parameter, $\mathrm{pK}_{\mathrm{A}}$ and $\log P$ values of the solutes

\begin{tabular}{|c|c|c|c|c|c|c|c|c|}
\hline No. & Solute name & $\begin{array}{l}\text { Hydrogen } \\
\text { bond } \\
\text { acidity } \\
\left(\alpha_{2}\right)^{\mathrm{a}}\end{array}$ & $\begin{array}{l}\text { Hydrogen } \\
\text { bond } \\
\text { basicity } \\
\left(\beta_{2}\right)^{\mathrm{a}}\end{array}$ & $\begin{array}{l}\text { Dipolarity } \\
\left(\pi_{2}\right)^{\mathrm{a}}\end{array}$ & $\begin{array}{l}\text { Characteristic } \\
\text { McGovan } \\
\text { volume }(V)^{\mathrm{a}}\end{array}$ & $\mathrm{pK}_{\mathrm{A}}^{\mathrm{b}}$ & $\begin{array}{l}\log P \\
{(\text { experimental })^{\mathrm{c}}}^{\mathrm{c}}\end{array}$ & $\begin{array}{l}\log P \\
(\text { simulated })^{\mathrm{c}}\end{array}$ \\
\hline 1 & Benzoic acid & 0.59 & 0.40 & 0.90 & 0.932 & 4.20 & 1.87 & 1.72 \\
\hline 2 & Phenol & 0.60 & 0.30 & 0.89 & 0.775 & 9.99 & 1.46 & 1.39 \\
\hline 3 & Ferulic acid & 0.85 & 0.87 & 1.46 & 1.428 & 4.52 & 1.51 & 1.58 \\
\hline 4 & Hydrocaffeic acid & 1.35 & 0.87 & 1.47 & 1.330 & 4.56 & & 1.04 \\
\hline 5 & Caffeic acid & 1.35 & 0.93 & 1.57 & 1.287 & 4.43 & 1.15 & 1.67 \\
\hline 6 & o-Coumaric acid & 1.07 & 0.79 & 1.39 & 1.229 & 4.61 & 1.46 & 1.74 \\
\hline 7 & p-Coumaric acid & 1.07 & 0.79 & 1.39 & 1.229 & 4.36 & 1.46 & 1.74 \\
\hline 8 & 4-Hydroxybenzoic acid & 1.00 & 0.72 & 1.29 & 0.990 & 4.48 & 1.58 & 1.58 \\
\hline 9 & Protocatechuic acid & 1.27 & 0.86 & 1.46 & 1.050 & 4.48 & 0.86 & 1.32 \\
\hline 10 & Sinapinic acid & 0.73 & 0.96 & 1.52 & 1.630 & 4.47 & & 1.63 \\
\hline 11 & Syringic acid & 0.67 & 0.89 & 1.41 & 1.380 & 4.34 & 1.04 & 1.55 \\
\hline 12 & Vanillic acid & 0.78 & 0.80 & 1.35 & 1.190 & 4.42 & 1.43 & 1.7 \\
\hline 13 & $\alpha$-Resorcylic acid & 1.56 & 0.93 & 1.48 & 1.049 & 3.94 & 0.86 & 1.29 \\
\hline 14 & Veratric acid & 0.57 & 0.90 & 1.68 & 1.330 & 4.44 & 1.61 & 1.52 \\
\hline
\end{tabular}

${ }^{a}$ Absolv software (http://pharma-algorithms.com/webboxes/)

${ }^{\mathrm{b}}$ From literature (Buszewski et al. 2006; Erdemgil et al. 2007)

${ }^{\mathrm{c}}$ ALOGPS 2.1 software (http://www.vcclab.org/lab/alogps/start.html)

Hold-up volume of the column was determined by injection of pure water into the column of every modifier system.

\section{Results and discussion}

In Figs. $1-3$ the values of retention $(\log k, k$ is retention factor) of investigated phenolic acids are correlated for $\mathrm{C} 18$ stationary phase (Zorbax SB C-18) in the systems with buffer mobile phases as follows $18 \%$ tetrahydrofuran vs. $12 \%$ acetonitrile, $18 \%$ tetrahydrofuran vs. $24 \%$ methanol and $12 \%$ acetonitrile vs. $24 \%$ methanol, respectively. The concentration values of the mobile phase modifiers were chosen on the basis of similar average retention and retention range of the solutes in the liquid chromatography systems investigated. The investigated phenolic acids (Table 2) demonstrate relatively high hydrophilic properties. Their retention values, $k$, are mainly in the range 1-10 for relatively low concentration of each modifier applied in the mobile phase.

In addition it is worthwhile to underline that concentration of the organic modifiers in the eluents is considerably smaller than that in the liquid phases of gas-liquid partition systems applied for correlation of partition constants of benzene derivatives mentioned above (Dzido 2000). Hence, the hydrophobic property of the all mobile phases is substantially stronger. It implies that participation of the modifier type to possible changes of separation selectivity by molecular interactions of the solute in the mobile phase is con- siderably diminished in comparison to that in liquid phases of partition systems mentioned. In this case, application of our approach to explanation the selectivity changes seems to be even more reasonable than that for the systems with higher concentration of the modifiers, what was previously reported (Dzido et al. 2002; Klimek-Turek et al. 2008).

\subsection{Tetrahydrofuran vs. acetonitrile}

In Figs. 1a-1c the correlations of $\log k$ values of phenolic acids for the mobile phases $18 \%$ tetrahydrofuran vs. $12 \%$ acetonitrile in mixtures with buffers of $\mathrm{pH} 3.0,4.6$ and 5.0 are presented, respectively. In these figures it is clearly seen that average retention of the solutes in the system with THF more strongly increases with $\mathrm{pH}$ decrease of buffer than that with ACN. This effect can be explained by stronger molecular interactions of the solutes and modifier molecules in the stationary phase region in system with THF than with ACN one. Dissociation of phenolic acids with respect to carboxy group is decreased in solution of lower $\mathrm{pH}$ value what leads to their stronger hydrophobic interactions in the stationary phase with THF than with $\mathrm{ACN}$ (compare $\mathrm{pK}_{\mathrm{A}}$ values of solutes in Table 2). The stationary phase in system with THF is more hydrophobic than with ACN due to stronger adsorption of the former modifier and its properties mentioned above.

Relative retention changes of syringic, vanillic and protocatechuic acids can also support the explanation of this 


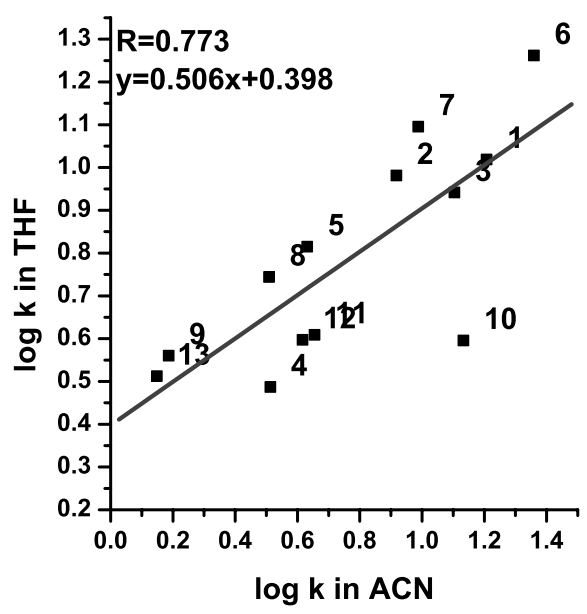

Fig. 1a $\log k$ in $18 \% \mathrm{v} / \mathrm{v}$ THF plotted against $\log k$ in $12 \% \mathrm{v} / \mathrm{v} \mathrm{ACN}$; $\mathrm{C} 18$ stationary phase, $\mathrm{pH}$ 3.0. Solute numbers as in Table 2

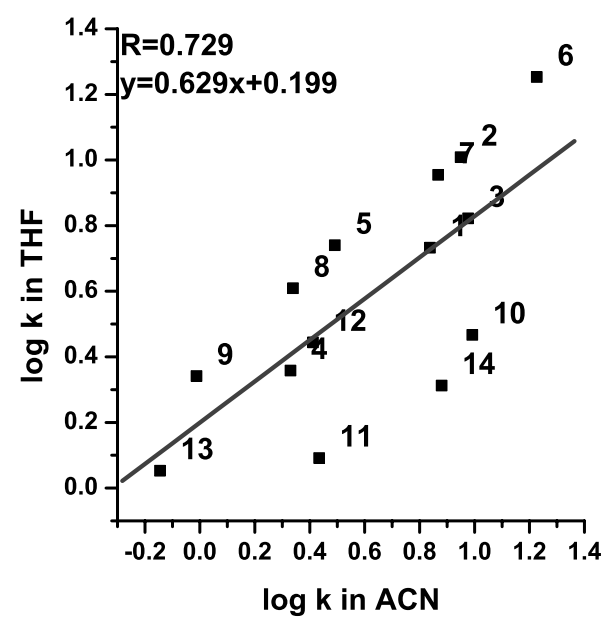

Fig. 1b $\log k$ in $18 \%$ v/v THF plotted against $\log k$ in $12 \%$ v/v ACN; $\mathrm{C} 18$ stationary phase, $\mathrm{pH}$ 4.6. Solute numbers as in Table 2

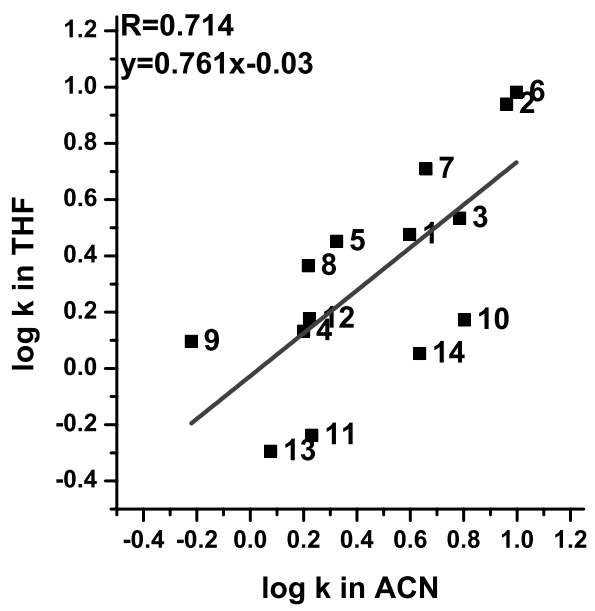

Fig. 1c $\log k$ in $18 \%$ v/v THF plotted against $\log k$ in $12 \%$ v/v ACN; C18 stationary phase, $\mathrm{pH}$ 5.0. Solute numbers as in Table 2 effect. These solutes demonstrate comparable retention in the THF system with the buffer of $\mathrm{pH} 3.0$. When $\mathrm{pH}$ of buffer in the mobile phase increases then retention of syringic acid decreases relative to vanillic and protocatechuic acids in THF system in comparison to ACN one. Syringic acid demonstrates the lowest value of $\mathrm{pK}_{\mathrm{A}}$ (Table 2) among the phenolic acids discussed. So the former solute is dissociated to higher degree in comparison to vanillic and protocatechuic acids in systems of $\mathrm{pH} 4.6$ and 5.0. Under such conditions and when stationary phase in THF system is more hydrophobic than in ACN one, then the mentioned changes of separation selectivity of syringic acid relative to vanillic acid or protocatechuic acid are reasonable.

The discussed effect is additionally confirmed by $\log k$ values of phenol. The phenol retention is not practically changed with $\mathrm{pH}$ variation of buffer in the mobile phase$\log k$ values of phenol are close to 1.0, contrary to phenolic acids, see Figs. 1a-1c. Phenol molecules are not dissociated in the $\mathrm{pH}$ range investigated. This implies its molecule is characterized by constant ability to H-bond formation and hydrophobic interactions with components (especially modifier) of the stationary phase irrespective of $\mathrm{pH}$ values in the range investigated.

Influence of solute ability to hydrogen bond interactions on retention and selectivity of phenolic acids is well pronounced in correlation graphs (Figs. 1a-1c). The points for phenolic acids (caffeic acid, o-coumaric acid, p-coumaric acid, 4-hydroxybenzoic acid, protocatechuic acid), which have large value of $\alpha_{2}$ parameter (Table 2), are positioned above the correlation line for all solutes. This effect is explained by stronger molecular interactions of these solutes, in comparison to the solutes of lower values of $\alpha_{2}$ parameter, with modifier in the stationary phase region in system with THF relative to that in system with ACN.

Points of phenolic acids, which have higher values of $\beta_{2}$ parameter (Table 2) and relatively small value of $\alpha_{2}$ parameter, lay below the correlation lines. It means the solutes show increase of retention relative to other phenolic acids in the ACN system in comparison to that of the THF one. This effect can be explained by proton acceptor-proton donor interactions between the solutes and acetonitrile molecules in the stationary phase. This interpretation seems to be reasonable because acetonitryle shows weak hydrogen bond acidity contrary to tetrahydrofuran, which does not demonstrate any proton donor property.

Characteristic relative retention changes are demonstrated by $\alpha$-resorcylic acid of which hydrogen bond acidity parameter is the largest one among the solutes investigated (Table 2). The point of this solute is positioned above the correlation line in Fig. 1a (pH of buffer in the mobile phase 3.0) what is in agreement with discussion above. However, in Figs. 1b (buffer $\mathrm{pH} 4.6$ ) and 1c (buffer $\mathrm{pH}$ 5.0) the point of $\alpha$-resorcylic acid is shifted underneath the correlation line. It should be mentioned that this shifting effect 
increases with $\mathrm{pH}$ increase. It could seem that this effect is not in agreement with the discussion above. Nevertheless, $\alpha$-resorcylic acid shows the lowest value of $\mathrm{pK}_{\mathrm{A}}$ (close to 4.0) amongst phenolic acids investigated (Table 2), what can be applied to explain this effect as follows. When $\mathrm{pH}$ of buffer in the mobile phase increases then resorcylic acid is considerably more dissociated than remaining phenolic acids. Then its retention begins more strongly to decrease in comparison to remaining phenolic acids in the THF system than in $\mathrm{ACN}$ one (stationary phase with tetrahydrofuran is more hydrophobic than with acetonitrile). This effect is reflected in shifting the point of resorcylic acid beneath the correlation line.

The changes of separation selectivity of solutes caused by their different ability to molecular interactions as proton donor with organic modifier in the stationary phase leads to variation of retention order in systems with $\mathrm{ACN}$ in comparison to that with THF. It is especially well pronounced for systems with buffer of $\mathrm{pH} 4.6$ or 5.0. In the system with acetonitrile the retention decrease of some phenolic acids is as follows: veratric, syringic, vanillic, 4-hydroxybenzoic and protocatechuic. Similar retention order is in the system with buffer $\mathrm{pH} 3.0$ (with exception of veratric acid of which retention data was not registered). Hydrophobic character of these compounds decreases in the same order (veratric acid posses two methoxy groups, syringic acid posses two metoxy and one hydroxy groups, vanillic acid one methoxy group and one hydroxy group, 4-hydroxybenzoic one hydroxy group and protocatechuic two hydroxy groups). Values of solvation energy of these solutes form the same order: $-38.004,-41.235,-45.507,-58.852$ and $-58.609 \mathrm{~J} / \mathrm{mol}$, respectively [PC Spartan Pro 1.0.5], compare also $\log P$ values in Table 2. Hence, the retention decrease of these solutes is in accordance with decrease of their hydrophobic character. However, in the THF system the order of retention is quite different. Retention of these phenolic acids in this system increases as follows: syringic, veratric, protocatechuic, vanillic, 4-hydroxybenzoic. This retention order can not be predicted basing on hydrophobic character of the solutes. In THF system the highest retention is demonstrated by 4hydroxybenzoic acid. This relative retention increase of the solute in THF system in comparison to ACN one can be explained by stronger participation of hydrogen bond interaction of this proton donor molecule with THF in the stationary phase than that with ACN (due to higher sorption of THF and its considerably stronger ability to hydrogen bond formation as proton acceptor relative to $\mathrm{ACN}$ ). In addition 4-hydroxybenzoic acid demonstrates relatively small molecular volume what enables its somewhat stronger entropic penetration the stationary phase region relative to remaining solutes. Veratric and syringic acids show the lowest relative retention in THF system among the solutes considered. This effect can be explained in respect of their the smallest $\mathrm{pK}_{\mathrm{A}}$ values. In addition these solutes show relatively larger molecular volume and branched molecular structure in comparison to vanillic, 4-hydroxybenzoic and protocatechuic acids what can lead to their somewhat lower entropic penetration the stationary phase in system with THF than with ACN. In addition, veratric and syringic acids demonstrate low values of $\alpha_{2}$ parameter, which is responsible for lower participation to H-bond formation with THF in the stationary phase region.

Another example of retention decrease in ACN system (pH 4.6 and 5.0), according to solvation energy decrease, is presented by the following order of solutes: sinapinic acid $(-47.129 \mathrm{~J} / \mathrm{mol})$ ferulic acid $(-48.578 \mathrm{~J} / \mathrm{mol})$, p-coumaric acid $(-55.954 \mathrm{~J} / \mathrm{mol})$, caffeic acid $(-61.916 \mathrm{~J} / \mathrm{mol})$. On the other hand, in the system with THF lower retention is observed for solutes of larger and more branched molecules. The retention of sinapinic acid in THF system is the distinguish example. This molecule has two methoxy groups. Considerably larger retention of this solute is observed in ACN system in comparison to that of THF one. Probable explanation is concerned with its relatively more branched molecular structure and small value of $\alpha_{2}$ parameter.

Stronger ordering of the stationary phase in the system with THF and ability of this modifier to form hydrogen bonds as the proton acceptor are responsible for changes of separation selectivity of solutes in tetrahydrofuran system relative to the systems with modifiers, which do not show these properties.

Syringic, vanillic and 4-hydroxybenzoic acids demonstrate similar retention in ACN system. 4-hydroxybenzoic acid is more hydrophilic than vanillic acid and much more than syringic acid. The last solute shows larger ability to dipolar interactions than the second one and considerably larger than the former solute. These properties of the three solutes can be used for explanation their relative retention changes. In the system with THF the retention of vanillic acid decreases and increases relative to 4-hydroxybenzoic acid and syringic acid, respectively, in comparison to ACN system. The mentioned solute properties enable to enhance the values of their separation factor in THF system in comparison to $\mathrm{ACN}$ one.

Analogous effect can be observed for cinnaminic acid derivatives. Separation selectivity of p-coumaric, ferulic and sinapinic acids in THF system is considerably higher than that in ACN one. On the other hand, the separation selectivity of ferulic and caffeic acids in THF system is lower than that in ACN one.

\subsection{Methanol vs. tetrahydrofuran}

In Figs. 2a-2c the correlations of $\log k$ values of the solutes for $18 \%$ tetrahydrofuran vs. $24 \%$ methanol with buffers of $\mathrm{pH}$ 3.0, 4.6 and 5.0 in the mobile phase are presented, respectively. It is seen that average retention of the solutes in 


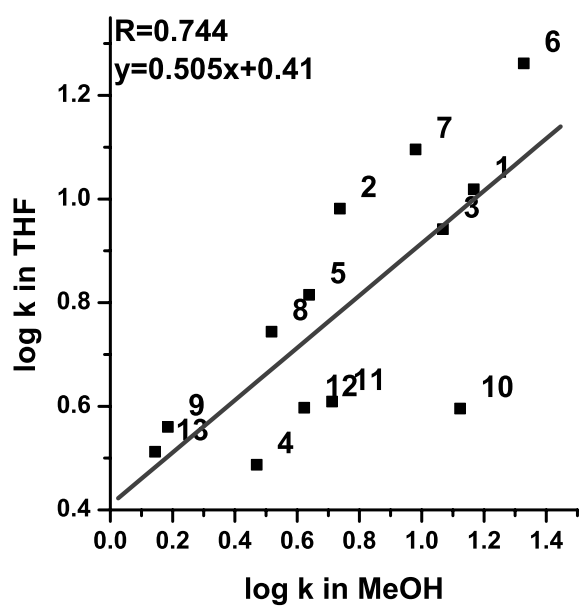

Fig. 2a $\log k$ in $18 \% \mathrm{v} / \mathrm{v}$ THF plotted against $\log k$ in $24 \% \mathrm{v} / \mathrm{v}$ $\mathrm{MeOH} ; \mathrm{C} 18$ stationary phase, $\mathrm{pH}$ 3.0. Solute numbers as in Table 2

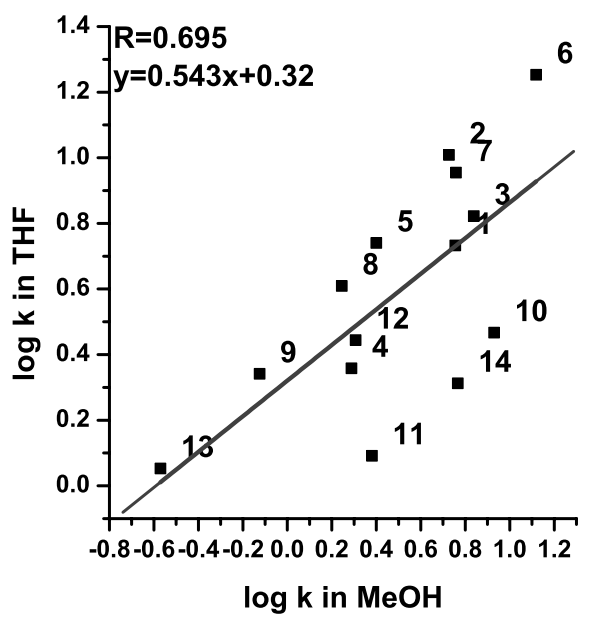

Fig. 2b $\log k$ in $18 \% \mathrm{v} / \mathrm{v}$ THF plotted against $\log k$ in $24 \% \mathrm{v} / \mathrm{v}$ $\mathrm{MeOH} ; \mathrm{C} 18$ stationary phase, $\mathrm{pH} 4.6$. Solute numbers as in Table 2

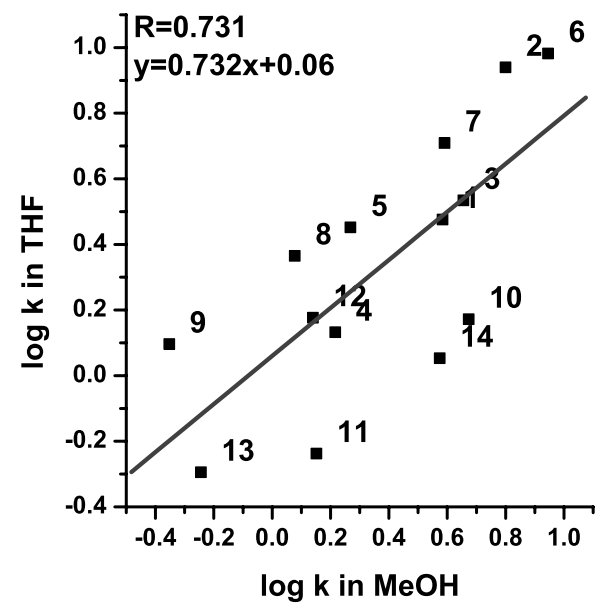

Fig. 2c $\log k$ in $18 \%$ v/v THF plotted against $\log k$ in $24 \%$ v/v MeOH; C18 stationary phase, $\mathrm{pH}$ 5.0. Solute numbers as in Table 2 the systems with THF increases more strongly with $\mathrm{pH}$ decrease of buffer than that with $\mathrm{MeOH}$. This effect should be rather attributed to considerably stronger hydrophobic character of the stationary phase in the system with THF than with $\mathrm{MeOH}$, compare discussion above for the systems THF vs. ACN. To some extent this effect could be diminished by participation of specific molecular interactions of solute and modifier in the stationary phase. Both modifiers can form H-bonds with proton donor solutes such as phenolic acids investigated. At lower $\mathrm{pH}$ these solutes tend to be less dissociated. In this way carboxy group of phenolic acids can participate in stable H-bond formation as proton donor, especially with $\mathrm{MeOH}$ (Alam and Callis 1994). However, concentration of methanol in the stationary phase region of $\mathrm{MeOH}$ system is considerably smaller than that of THF system (McCormick and Karger 1980; Gritti et al. 2007). The discussed effect is additionally confirmed by the retention data of phenol. The phenol retention does not change with $\mathrm{pH}$ of buffer in the mobile phase of both systems $\left(\log k_{\mathrm{MeOH}} \approx 0.75 ; \log k_{\mathrm{THF}} \approx 1.00\right)$, compare also discussion above for the systems THF vs. ACN.

All solutes with unshielded hydroxy group in their molecules (caffeic acid, 4-hydroxybenzoic acid, protocatechuic acid, $\alpha$-resorcylic acid) and phenol practically demonstrate retention increase relative to remaining solutes (with shielded hydroxy group e.g. ferulic acid, syringic acid, vanillic acid) in THF system in comparison to $\mathrm{MeOH}$ one. This effect is reflected in position of points of the former group of solutes those lay above the correlation line. These solutes show higher values of hydrogen bond acidity parameter than the solutes, of which points lays below or on correlation line, compare values of solvation parameters in Table 2. As it has been stressed above, stronger hydrogen bond interactions of the solutes, showing higher values of $\alpha_{2}$ parameter, with THF in the stationary phase are responsible for this effect.

Methanol is also able to interact by hydrogen bonds with proton donor solutes. However, this modifier demonstrates considerably lower extraction into the stationary phase region than tetrahydrofuran. So the methanol effect on relative retention increase of phenolic acids, by molecular interactions in the stationary phase, seems to be substantially lower than that of tetrahydrofuran.

Particular separation selectivity changes presented by the tetrahydrofuran and methanol systems are very similar to those discussed above for the THF and ACN systems. This remark is also confirmed by the data presented in the next section where phenolic acid retention is compared for $\mathrm{MeOH}$ and $\mathrm{ACN}$ systems.

\subsection{Acetonitrile vs. methanol}

As it is seen in Figs. 3a-3c, correlations of phenolic acid retention for $24 \% \mathrm{MeOH}$ and $12 \% \mathrm{ACN}$ systems with buffer 


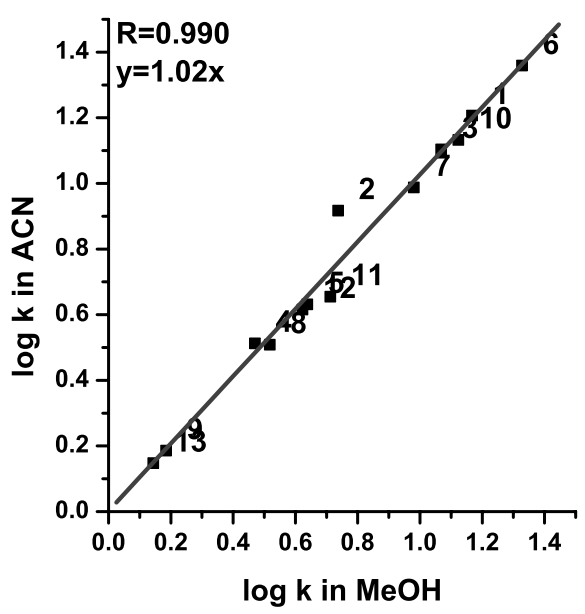

Fig. 3a $\log k$ in $12 \% \mathrm{v} / \mathrm{v}$ ACN plotted against $\log k$ in $24 \% \mathrm{v} / \mathrm{v}$ $\mathrm{MeOH}$; $\mathrm{C} 18$ stationary phase, $\mathrm{pH}$ 3.0. Solute numbers as in Table 2

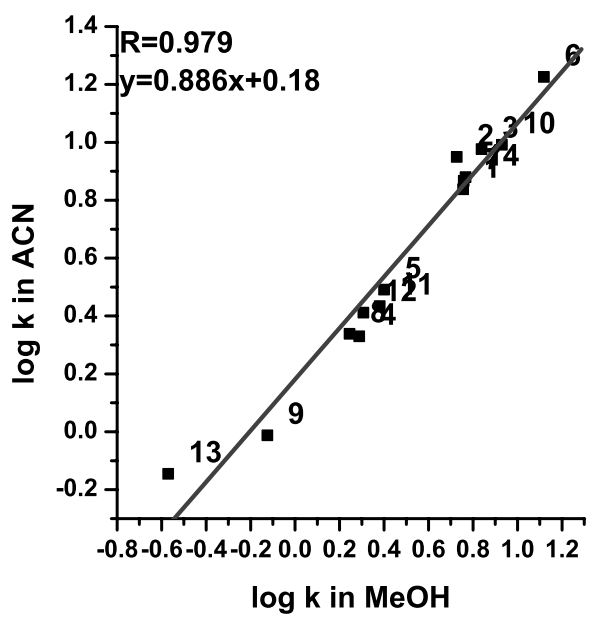

Fig. 3b $\log k$ in $12 \% \mathrm{v} / \mathrm{v}$ ACN plotted against $\log k$ in $24 \% \mathrm{v} / \mathrm{v}$ $\mathrm{MeOH}$; $\mathrm{C} 18$ stationary phase, $\mathrm{pH}$ 4.6. Solute numbers as in Table 2

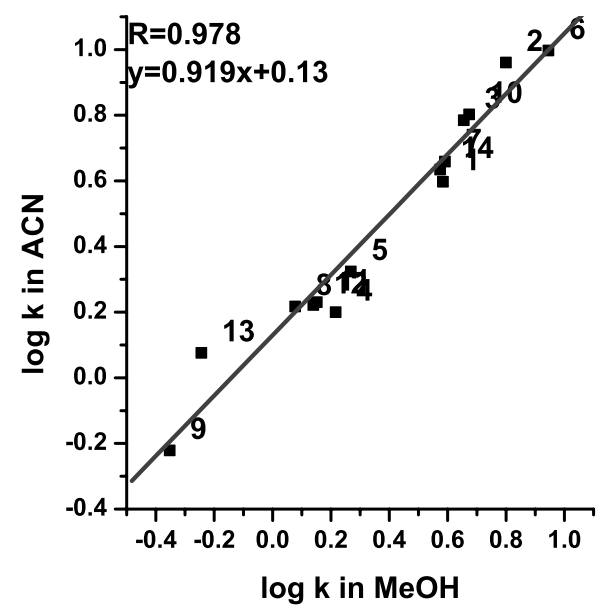

Fig. 3c $\log k$ in $12 \%$ v/v ACN plotted against $\log k$ in $24 \% \mathrm{v} / \mathrm{v}$ $\mathrm{MeOH}$; $\mathrm{C} 18$ stationary phase, $\mathrm{pH}$ 5.0. Solute numbers as in Table 2
pH 3.0, 4.6 and 5.0, respectively, show very high values of correlation coefficient. It means separation selectivity of phenolic acids in these systems is very similar. This effect is probably concerned with properties of the modifier molecules. Methanol and acetonitrile show comparable molecular volume and demonstrate relatively lower extraction into the stationary phase in comparison to tetrahydrofuran. Nevertheless. acetonitrile shows higher extraction than methanol, however, concentration of the former modifier in the mobile phase is half of that of methanol. It implies that concentration of both modifiers in the stationary phase of their chromatographic systems can be comparable. Under such conditions influence of molecular interactions of the solutes with modifier in the stationary phase can lead to minor differentiation of separation selectivity of phenolic acids in these systems. This effect is reflected by very good correlation of phenolic acid retention presented in Fig. 3.

We would like to draw readers' attention to two solutes: phenol and $\alpha$-resorcylic acid, which demonstrate subtle relative retention changes. The point of former solute lies slightly, but clearly, above the correlation line presented especially in Fig. 3a where systems with buffer of $\mathrm{pH} 3.0$ are compared. It means that phenol demonstrates retention increase relative to phenolic acids in ACN system in comparison to $\mathrm{MeOH}$ one. This effect can be also expressed as retention increase of phenolic acids relative to phenol in the methanol system in comparison to the acetonitrile one. The last sentence is more appropriate for explanation of this separation selectivity change. Phenolic acids are weakly or practically not dissociated in the mobile phase $(\mathrm{pH} 3.0)$. Then the carboxy group, under this condition ( $\mathrm{pH}$ 3.0) as proton donor group, of each phenolic acid molecule can take part in formation of stronger complexes with methanol molecules in the stationary phase (Alam and Callis 1994). When $\mathrm{pH}$ of buffer in the mobile phase increases then the solutes are ionized to higher degree what leads to diminution of formation of the mentioned complexes. This discussion is confirmed by slightly decreased distance of point location of phenol from correlation lines in Figs. $3 b$ and $3 c$ in comparison to that in Fig. 3a.

Analogous effect demonstrates $\alpha$-resorcylic acid. The point of this solute is located directly on the correlation line what indicates no selectivity change relative to remaining phenolic acids, Fig. 3a. However, retention of $\alpha$ resorcylic acid decreases relative to remaining phenolic acids in methanol system in comparison to acetonitrile one when $\mathrm{pH}$ of buffer in the mobile phase increases. This effect is reflected by position of $\alpha$-resorcylic acid point in Figs. 3b and $3 \mathrm{c}$ - this point is clearly above the correlation line. The explanation of this effect is as follows. $\alpha$-Resorcylic acid demonstrates the lowest value of $\mathrm{pK}_{\mathrm{A}}$ (close to 4.0) among the group of phenolic acids investigated, Table 2. It implies that at $\mathrm{pH} 3.0$ of buffer in the mobile phase all phenolic acids 
are practically as non-dissociated molecules and can form complexes with methanol in the stationary phase. At higher buffer $\mathrm{pH}$ (4.6) the degree of dissociation of phenolic acids is increased. However, dissociation of $\alpha$-resorcylic acid is the strongest among phenolic acids investigated. Under this condition the solute (due to the lowest value of $\mathrm{pK}_{\mathrm{A}}$ ) shows diminution of molecular interactions with methanol in the stationary phase in comparison to remaining phenolic acids of which $\mathrm{pK}_{\mathrm{A}}$ values are clearly higher.

\section{Conclusion}

The results presented in the paper confirm our previous assumption that explanation of separation selectivity (relative retention) changes in reversed phase systems with adsorbent of $\mathrm{C}-18$ type, when one modifier is replaced by another in the mobile phase, can be performed taking into account molecular interactions of the solutes with the stationary phase components, especially mobile phase modifier (organic solvent) extracted into the stationary phase. Molecular interactions of the solutes in the mobile phase can be then neglected. Such approach leads to simplified interpretation of separation selectivity changes because it takes into consideration molecular interactions in one phase only.

Open Access This article is distributed under the terms of the Creative Commons Attribution Noncommercial License which permits any noncommercial use, distribution, and reproduction in any medium, provided the original author(s) and source are credited.

\section{References}

Abraham, M.H., Chadha, H.S., Martins, F., Mitchell, R.C., Bradbury, M.W., Gratton, J.A.: Hydrogen bonding part 46: A review of the correlation and prediction of transport properties by an LFER method: physicochemical properties, brain penetration and skin permeability. Pestic. Sci. 55, 78-88 (1999)

Alam, M.K., Callis, J.B.: Elucidation of species in alcohol-water mixtures using near-IR spectroscopy and multivariate statistics. Anal. Chem. 66, 2293-2301 (1994)

Bidlingmeyer, B.A.: Separation of ionic compounds by reversed-phase liquid chromatography - an update of ion-pairing techniques. J. Chromatogr. Sci. 18, 525-539 (1980)

Bocian, S., Vajda, P., Felinger, A., Buszewski, B.: Excess adsorption of commonly used organic solvents from water on nonendcapped C18-bonded phases in reversed-phase liquid chromatography. Anal. Chem. 81, 6334-6346 (2009)
Buszewski, B., Kowalska, S., Karasov, G., Lehotay, J.: Influence of $\mathrm{pH}$ on benzoic acid derivatives' retention and RP HPLC column classification. J. Liq. Chrom. Rel. Technol. 29, 2663-2675 (2006)

Dzido, T.H.: Modifier influence on selectivity of reversed-phase HPLC systems. J. Liq. Chrom. Rel. Technol. 23, 2773-2788 (2000)

Dzido, T.H., Kossowski, T.E., Matosiuk, D.: Comparison of retention of aromatic hydrocarbons with polar groups in binary reversedphase high-performance liquid chromatography systems. J. Chromatogr. A 947, 167-183 (2002)

Erdemgil, F.Z., Şanli, S., Şanli, N., Özkan, G., Barbosa, J., Guiteras, J., Beltrán, J.L.: Determination of pKa values of some hydroxylated benzoic acids in methanol-water binary mixtures by LC methodology and potentiometry. Talanta 72, 489-496 (2007)

Gilpin, R.K., Jaroniec, M., Lin, S.: Characterization of the surface composition of alkyl bonded phases under reversed-phase liquid chromatographic conditions using homologues of alkanoate and perfluoroalkanoate esters as solute probes. Anal. Chem. 62, 20922098 (1990)

Gritti, F., Kazakevich, Y.V., Guiochon, G.: Effect of the surface coverage of endcapped C18-silica on the excess adsorption isotherms of commonly used organic solvents from water in reversed phase liquid chromatography. J. Chromatogr. A 1169, 111-124 (2007)

Karapetian, Y.A., Eichis, W.N.: Physical Chemistry Properties of Electrolytes in Non-Aqueous Solutions. Khimia, Moscow (1989)

Kazakevich, Y.V., LoBrutto, R., Chan, F., Patel, T.: Interpretation of the excess adsorption isotherms of organic eluent components on the surface of reversed phase adsorbents: Effect on the analyte retention. J. Chromatogr. A 913, 75-87 (2001)

Kiridena, W., Poole, C.F.: Structure-driven retention model for solvent selection and optimization in reversed-phase thin-layer chromatography. J. Chromatogr. A 802, 335-347 (1998)

Klimek-Turek, A., Dzido, T.H., Engelhardt, H.: The effect of modifier on selectivity in reversed-phase high performance liquid chromatography. LC.GC Europe 21, 33-42 (2008)

Lavine, B.K., Ritter, J.P., Peterson, S.: Enhancement of selectivity in reversed-phase liquid chromatography. J. Chromatogr. A 946, 8390 (2002)

McCormick, M., Karger, B.L.: Distribution phenomena of mobilephase components and determination of dead volume in reversedphase liquid chromatography. Anal. Chem. 52, 2249-2257 (1980)

Sandi, A., Szepesy, A.: Evaluation and modulation of selectivity in reversed-phase high-performance liquid chromatography. J. Chromatogr. A 845, 113-131 (1999)

Slaats, E.H., Markowski, W., Fekete, J., Poppe, H.: Distribution equilibria of solvent components in reversed-phase liquid chromatographic columns and relationship with the mobile phase volume. J. Chromatogr. 207, 299-323 (1981)

Szepesy, L.: Effect of molecular interactions on retention and selectivity in reversed-phase liquid chromatography. J. Chromatogr. 960, 69-83 (2002)

Tan, L.C., Carr, P.W., Abraham, M.H.: Study of retention in reversedphase liquid chromatography using linear solvation energy relationships I. The stationary phase. J. Chromatogr. A 752, 1-18 (1996) 